\title{
Prevalence and Correlates of Depressive Symptoms among Medical Students: a Cross- sectional Single-centre Study
}

\author{
M Al-Maashani, N Al-Balushi, M Al-Alawi, H Mirza, S Al-Huseini, M Al-Balushi, Y Obeid, S Jose, \\ N Al-Sibani, S Al-Adawi
}

\begin{abstract}
Background: Depressive symptoms are common among medical students. The aim of the present study was to determine the prevalence and risk factors of depressive symptoms among medical students in Sultan Qaboos University in Oman.

Method: A cross-sectional study was conducted among a random sample selected from 1041 medical students at Sultan Qaboos University, Oman. The Patient Health Questionnaire-9 (PHQ-9) was used to screen for depressive symptoms. A logistic regression model was used to determine risk factors for depressive symptoms.

Results: Of 197 medical students selected, 189 (61 men and 128 women) responded. The PHQ-9 results showed that the prevalence of depressive symptoms was $41.3 \%$. In multivariate analysis, female students were more likely than male students to develop depression (adjusted odds ratio $=2.866, \mathrm{p}=0.004$ ) . Medical students with a family history of depression were more likely to develop depression than those without a family history of depression (adjusted odds ratio $=4.150, \mathrm{p}=0.014$ ).

Conclusion: Depressive symptoms are common among medical students in Sultan Qaboos University. Risk factors for depressive symptoms are female sex and family history of depression.
\end{abstract}

Key words: Depression; Oman; Patient Health Questionnaire; Students, medical

Mohammed Al-Maashani, MD, Psychiatry Residency Training Program, Oman Medical Specialty Board, Muscat, Oman

Naser Al-Balushi, MD, Psychiatry Residency Training Program, Oman Medical Specialty Board, Muscat, Oman

Mohammed Al-Alawi, MD, Psychiatry Residency Training Program, Oman Medical Specialty Board, Muscat, Oman; Department of Behavioral Medicine, College of Medicine \& Health Sciences, Sultan Qaboos University, Muscat, Oman

Hassan Mirza, MD, MSc, Department of Behavioral Medicine, College of Medicine \& Health Sciences, Sultan Qaboos University, Muscat, Oman

Salim Al-Huseini, MD, Psychiatry Residency Training Program, Oman Medical Specialty Board, Muscat, Oman

Manal Al-Balushi, MD, Psychiatry Residency Training Program, Oman Medical Specialty Board, Muscat, Oman

Yousif Obeid, MD, Department of Behavioral Medicine, College of Medicine \& Health Sciences, Sultan Qaboos University, Muscat, Oman

Sachin Jose, MSc, Research and Statistics, Oman Medical Specialty Board, Muscat, Oman

Nasser Al-Sibani, Department of Behavioral Medicine, College of Medicine \& Health Sciences, Sultan Qaboos University, Muscat, Oman

Samir Al-Adawi, MD, Department of Behavioral Medicine, College of Medicine \& Health Sciences, Sultan Qaboos University, Muscat, Oman

Address for correspondence: Dr Naser Al Balushi, Psychiatry Residency Training Program, Oman Medical Specialty Board, Muscat, Sultanate of Oman. Email: naser.albalushi@hotmail.com

Submitted: 25 March 2018; Accepted: 26 March 2019

\section{Introduction}

Psychological distress among college students is increasingly recognised. ${ }^{1}$ However, depressive symptoms among medical students often go unrecognised, compared to the general population. ${ }^{2}$ This 'silent epidemic' has been reported in medical schools in Gulf Cooperation Council countries. ${ }^{3} \mathrm{~A}$ meta-analysis reported that the prevalence of depressive symptoms among medical students in Gulf Cooperation Council countries was higher than the international trend. ${ }^{4}$ Moreover, these medical students have the highest dropout rate. ${ }^{5}$ In Oman, approximately $17 \%$ of medical students have depressive symptoms. ${ }^{6}$ The present study aimed to determine the prevalence and risk factors of depressive symptoms among medical students in Sultan Qaboos University in Oman.

\section{Methods}

This study was approved by the Ethics Committee of the College of Medicine at Sultan Qaboos University Hospital (MREC\#893) and was conducted from August to December 2015. At the time of the study, there were 1041 medical students from all six academic years at the College of Medicine and Health Sciences of Sultan Qaboos University. ${ }^{7}$

Based on the $17 \%$ prevalence of depression among medical students in Sultan Qaboos University, ${ }^{6}$ the minimum sample size to have a power level of $80 \%$ was calculated as 171 , with a type- 1 error of $5 \%$ (alpha $=0.05)$ and significance level of $95 \%$. Students were selected at random using a software randomizer; a sample of 197 was generated to allow for non-responses. Because stress level may differ among medical student year-groups, ${ }^{8}$ the present 
cohort was categorised as either pre-clinical (years 1 to 4 ) or clinical (years 5 to 6 ).

The outcome measure was the self-administered Patient Health Questionnaire-9 (PHQ-9). The PHQ-9 contains items adopted from the DSM-IV and aids in the diagnosis of depressive symptoms and their severity. It has been psychometrically validated in different settings (including primary, secondary, and tertiary), cultures, and samples. Its performance was comparable to that of doctoradministered scales in diagnosing depression. In a validation study in Oman, a cut-off score of $\geq 10$ had a likelihood ratio of 10 for a positive test result. ${ }^{9}$ In the present study, the same cut-off score was used for the presence of depressive symptoms. A total score of 10 to 14 was considered moderate depression, 15 to 19 moderately severe depression, and 20 to 27 severe depression, in accordance with the DSM-IV.

Those with or without depression were compared using Chi-squared test or Fisher's exact test for categorical variables. Associations between sociodemographic and clinical variables and the presence of depression were determined using univariate and multivariate analyses. Variables associated with depression in the univariate analysis were entered into a stepwise backward logistic regression model to determine the correlates of depression and to adjust for confounders. A two-tailed significance level was set at 0.05. Statistical analysis was carried out using SPSS (Windows version 22.0; IBM Corp, Armonk [NY], United States). ${ }^{10}$

\section{Results}

Of 197 medical students selected, 189 (61 men and 128 women) responded (Table 1). Of them, $94 \%$ were single; $75.7 \%$ were preclinical students and $24.3 \%$ were clinical students. $93.7 \%$ has never diagnosed with depressive disorder. $90.5 \%$ had no family history of depressive disorder. $97.9 \%$ has never used alcohol or opiates. According to PHQ-9 results, the prevalence of depressive symptoms was $41.3 \%$.

In univariate analysis, female sex $(\mathrm{p}=0.01)$, previous diagnosis with depression $(\mathrm{p}=0.03)$, and a family history of depression (0.01) were risk factors for depression among medical students (Table 2).

In multivariate analysis, female students were more likely to develop depression than male students (adjusted odds ratio $=2.866,95 \%$ confidence interval $=1.403-5.854$, $\mathrm{p}=0.004$, Table 3 ). Medical students with a family history of depression were more likely to develop depression than those without a family history of depression (adjusted odds ratio $=4.150,95 \%$ confidence interval $=1.335-12.905, \mathrm{p}=$ 0.014 , Table 3).

\section{Discussion}

In a meta-analysis of 167 studies from 43 countries involving 116628 people, the prevalence of depression and depressive symptoms was $27.2 \% .{ }^{8}$ In Oman, the prevalence has ranged
Table 1. Sociodemographic variables of medical students $(n=189)$

\begin{tabular}{|lc|}
\hline Variable & $\begin{array}{c}\text { No. (\%) of } \\
\text { participants }\end{array}$ \\
Sex & $61(32.3)$ \\
Male & $128(67.7)$ \\
Female & $179(94.7)$ \\
\hline Marital status & $8(4.2)$ \\
Single & $2(1.1)$ \\
Married & \\
Divorced & $62(32.8)$ \\
\hline Residence & $89(47.1)$ \\
Living with family & $38(20.1)$ \\
Staying on campus & \\
Living in a rented apartment / house & $143(75.7)$ \\
\hline Year of study & $46(24.3)$ \\
Pre-clinical & \\
Clinical & \\
\hline Have you ever diagnosed with & $177(93.7)$ \\
depressive disorder? & $12(6.3)$ \\
No & \\
Yes & $171(90.5)$ \\
\hline Do you have a family history of & $18(9.5)$ \\
depression disorder? & \\
No & \\
Yes & \\
\hline Have you ever used substances such as & \\
alcohol and opiates? & \\
No & \\
Yes & \\
\hline
\end{tabular}

from $11.4 \%{ }^{6}$ to $27.7 \% .^{7}$ In Saudi Arabia, the prevalence was $46.2 \%$ to $48.2 \% .{ }^{11}$ In United Arab Emirates, the prevalence was $28.6 \% .{ }^{12}$ In Bahrain, $19 \%{ }^{13}$ to $40 \%{ }^{14}$ of medical student were reported to have depressive symptoms. In the present study, the prevalence of depressive symptoms was $41.3 \%$. The discrepancy could be due to case ascertainment bias, heterogeneity in the methodology, the healthcare system, and cultural differences. ${ }^{8}$ Arab culture discourages expression of emotion. The PHQ-9 is designed to solicit the 'cognitive' aspect of depression rather than 'somatic' types, which are rife as idiom of distress in Arab populations. Nonetheless, the present study indicated that depression is rife among medical students in terms of the PHQ-9, consistent with a meta-analysis of 77 studies involving 62728 medical students that suggested that depressive symptoms are more common among medical students in Gulf Cooperation Council countries than elsewhere. ${ }^{4}$

In multivariate analysis, female students had higher odds of having depressive symptoms, consistent with previous studies on the general and college populations. ${ }^{4,8}$ 
Table 2. Univariate analysis for risk factors of depression

\begin{tabular}{|c|c|c|c|}
\hline \multirow[t]{2}{*}{ Variable } & \multicolumn{2}{|c|}{ No. (\%) of participants } & \multirow[t]{2}{*}{ p Value } \\
\hline & No depression & Depression & \\
\hline Sex & & & 0.011 \\
\hline Male & $44(72.1)$ & $17(27.9)$ & \\
\hline Female & $67(52.3)$ & $61(47.7)$ & \\
\hline Marital status & & & 0.947 \\
\hline Single & $105(58.7)$ & $74(41.3)$ & \\
\hline Married & $5(62.5)$ & $3(37.5)$ & \\
\hline Divorced & $1(50.0)$ & $1(50.0)$ & \\
\hline Residence & & & 0.408 \\
\hline Living with family & $38(61.3)$ & $24(38.7)$ & \\
\hline Staying on campus & $48(53.9)$ & $41(46.1)$ & \\
\hline Living in a rented apartment / house & $25(65.8)$ & $13(34.2)$ & \\
\hline Year of study & & & 1.000 \\
\hline Pre-clinical & $84(58.7)$ & $59(41.3)$ & \\
\hline Clinical & $27(58.7)$ & $19(41.3)$ & \\
\hline Have you ever diagnosed with depressive disorder? & & & 0.030 \\
\hline No & $108(61.0)$ & $69(39.0)$ & \\
\hline Yes & $3(25.0)$ & $9(75.0)$ & \\
\hline Do you have a family history of depression disorder? & & & 0.010 \\
\hline No & $106(62.0)$ & $65(38.0)$ & \\
\hline Yes & $5(27.8)$ & $13(72.2)$ & \\
\hline Have you ever used substances such as alcohol and opiates? & & & 0.308 \\
\hline No & $110(59.5)$ & $75(40.5)$ & \\
\hline Yes & $1(25.0)$ & $3(75.0)$ & \\
\hline
\end{tabular}

Table 3. Multivariate analysis for predictors of depression (Patient Health Questionnaire-9 score of $\geq 10$ ).

\begin{tabular}{|lccc|}
\hline Variables & B & $\begin{array}{c}\text { Adjusted odds ratio } \\
\text { (95\% confidence interval) }\end{array}$ & p Value \\
$\begin{array}{l}\text { Sex } \\
\quad \text { Male (ref) }\end{array}$ & & & \\
$\quad$ Female & 1.053 & $2.866(1.403-5.854)$ & 0.004 \\
\hline $\begin{array}{l}\text { Have you ever diagnosed with depressive disorder? } \\
\quad \text { No (ref) }\end{array}$ & & & \\
$\quad$ Yes & 0.358 & $1.430(0.080-25.453)$ & 0.808 \\
\hline $\begin{array}{l}\text { Have you ever been prescribed treatment for a depressive } \\
\text { disorder? } \\
\quad \text { No (ref) }\end{array}$ & & & \\
$\quad$ Yes & 1.411 & $4.099(0.223-75.232)$ & 0.342 \\
\hline $\begin{array}{l}\text { Do you have a family history of depression disorder? } \\
\quad \text { No (ref) }\end{array}$ & & & \\
$\quad$ Yes & 1.423 & $4.150(1.335-12.905)$ & 0.01 \\
\hline
\end{tabular}

Family history of depression also plays a significant role in the development of depressive symptoms, consistent with a meta-analysis that supports temporal relationship between depressive symptoms and family history of depressive illness. ${ }^{15}$ In previous studies in Gulf Cooperation Council countries, determinants of depressive illness included 
burnout, year of study, sex, relationship with peers, and academic performance. ${ }^{11,16}$

This study has several limitations. First, the study was cross-sectional, so the results cannot be used to isolate the cause and effect. Second, self-administered checklists are inferior to gold-standard interview. Third, other factors were not explored such as socioeconomic status, contact with family and/or relatives, academic performance, history of resitting examinations or repeating, and history of self-harm. Future studies should use a more robust methodology, and other potentially modifiable factors should be investigated.

\section{Conclusion}

Depressive symptoms are common among medical students in Sultan Qaboos University. Risk factors for depressive symptoms are female sex and family history of depression.

\section{Declaration}

The authors have no conflict of interest to disclose. No financial support was received.

\section{References}

1. Hunt J, Eisenberg D. Mental health problems and help-seeking behavior among college students. J Adolesc Health 2010;46:310. Crossref

2. Dyrbye LN, Thomas MR, Shanafelt TD. Systematic review of depression, anxiety, and other indicators of psychological distress among U.S. and Canadian medical students. Acad Med 2006;81:35473. Crossref

3. Hamdy H, Telmesani AW, Wardy NA, Abdel-Khalek N, Carruthers G, Hassan F, et al. Undergraduate medical education in the Gulf Cooperation Council: a multi-countries study (Part 2). Med Teach 2010;32:290-5. Crossref

4. Puthran R, Zhang MW, Tam WW, Ho RC. Prevalence of depression amongst medical students: a meta-analysis. Med Educ 2016;50:45668. Crossref
5. Maher BM, Hynes H, Sweeney C, Khashan AS, O'Rourke M, Doran $\mathrm{K}$, et al. Medical school attrition-beyond the statistics a ten year retrospective study. BMC Med Educ 2013;13:13. Crossref

6. Al-Busaidi Z, Bhargava K, Al-Ismaily A, Al-Lawati H, Al-Kindi R, Al-Shafaee $\mathrm{M}$, et al. Prevalence of depressive symptoms among university students in Oman. Oman Med J 2011;26:235-9. Crossref

7. Albarwani S, Al-Saadoon M, Al-Rawas O, Al-Yaarubi S, Al-Abri R, Al-Lamki L, et al. Reflections on the academic accreditation of the MD programme of the College of Medicine \& Health Sciences, Sultan Qaboos University, Oman. Sultan Qaboos Univ Med J 2014;14:e7e11. Crossref

8. Rotenstein LS, Ramos MA, Torre M, Segal JB, Peluso MJ, Guille C, et al. Prevalence of depression, depressive symptoms, and suicidal ideation among medical students: a systematic review and metaanalysis. JAMA 2016;316:2214-36. Crossref

9. Al-Ghafri G, Al-Sinawi H, Al-Muniri A, Dorvlo AS, Al-Farsi YM, Armstrong K, et al. Prevalence of depressive symptoms as elicited by Patient Health Questionnaire (PHQ-9) among medical trainees in Oman. Asian J Psychiatr 2014;8:59-62. Crossref

10. IBM SPSS statistics for windows, version 20.0. 2013.

11. Al-Faris EA, Irfan F, Van der Vleuten CP, Naeem N, Alsalem A, Alamiri $\mathrm{N}$, et al. The prevalence and correlates of depressive symptoms from an Arabian setting: a wake up call. Med Teach 2012;34(Suppl 1):S326. Crossref

12. Ahmed I, Banu H, Al-Fageer R, Al-Suwaidi R. Cognitive emotions: depression and anxiety in medical students and staff. J Crit Care 2009;24:e1-7. Crossref

13. Abdelaziz AMY, Alotaibi KT, Alhurayyis JH, Alqahtani TA, Alghamlas AM, Algahtani HM, et al. The association between physical symptoms and depression among medical students in Bahrain. Int J Med Educ 2017;8:423-7. Crossref

14. Mahroon ZA, Borgan SM, Kamel C, Maddison W, Royston M, Donnellan C. Factors associated with depression and anxiety symptoms among medical students in Bahrain. Acad Psychiatry 2018;42:3140. Crossref

15. Sullivan PF, Neale MC, Kendler KS. Genetic epidemiology of major depression: review and meta-analysis. Am J Psychiatry 2000;157:155262. Crossref

16. Al-Alawi M, Al-Sinawi H, Al-Qubtan A, Al-Lawati J, Al-Habsi A, AlShuraiqi M, et al. Prevalence and determinants of burnout syndrome and depression among medical students at Sultan Qaboos University: a cross-sectional analytical study from Oman. Arch Environ Occup Health 2019;74:130-9. Crossref 